\title{
The Effects of the Random Transverse Crystal Field on the Spin-1 Model
}

\author{
E. AlbAYRAK* \\ Erciyes University, Department of Physics, 38039, Kayseri, Turkey \\ (Received August 8, 2014; in final form January 17, 2015)
}

The effects of random transverse crystal field are investigated in the mean-field approximation on the spin-1 model. The transverse crystal field is applied on a lattice in a bimodal random distribution with a tunable parameter adjusting the strength of one mode with respect to the other one. Then, the phase diagrams are calculated on the reduced temperature-transverse crystal field planes for given values of probability $p$ and coordination number $q$ at zero external magnetic field $h$. It was found that the model gives both second- and first-order phase transitions and tricritical points which is a well-known behavior of the spin-1 model. In addition, the model also yields critical end points and end points for the first-order phase transition lines for appropriate $p$ values.

DOI: $10.12693 /$ APhysPolA.127.818

PACS: $75.10 . \mathrm{Hk}, 75.30 . \mathrm{Kz}, 75.50 . \mathrm{Gg}$

\section{Introduction}

The existence of crystal field $(\mathrm{CF})$ in the spin-1 Ising model, called as the Blume-Capel (BC) model, leads to the first-order phase transitions and tricritical points (TCP). This is caused by the competition between the bilinear exchange interaction and $\mathrm{CF}$ which are uniaxial, i.e. both are along the $z$-axis. Instead of the uniaxial one, the $\mathrm{CF}$ along the $x$-axis, i.e. transverse to the bilinear exchange interaction parameter direction, is also very interesting, therefore, it has been getting a lot of attention.

The effects of transverse CF were investigated for different spin systems. One of the first works studies the structure of phase transitions for spin-one Heisenberg ferromagnet with uniaxial and biaxial single-ion anisotropy which was investigated within the mean-field approximation (MFA) [1]. The next one uses the MFA and random phase approximation to investigate the thermodynamic properties of spin-one Heisenberg ferromagnet with uniaxial and biaxial anisotropy [2]. The magnetic properties of spin-one Heisenberg ferromagnet with general crystal field potential were investigated by using the linked cluster expansion [3]. In addition to these, the ferromagnetic spin-1 Ising model with transverse CF was studied within the framework of effective field theory (EFT) on honeycomb, square, and cubic lattices [4]. The phase diagram of the spin-1 Ising model in the presence of a biaxial CF anisotropy was studied within the framework of a variational approach which was based on the Bogolyubov inequality for the free energy and where the effects of transverse $\mathrm{CF} D_{y}$ on the phase diagram in the $T-D_{x}$ plane were investigated [5]. The magnetic properties of spin-1 Ising model were studied with transverse $\mathrm{CF}$ and external magnetic field within the framework of the EFT with a probability distribution technique which accounts for

*corresponding author; e-mail: albayrak@erciyes.edu.tr the self-spin correlations. The influence of transverse CF on the phase diagrams, hysteresis loops and susceptibility was discussed [6]. A ferromagnetic spin-1 Ising model with transverse $\mathrm{CF}$ term was investigated by means of mapping to the usual spin- $1 / 2$ transverse Ising model and the location of the $T=0$ quantum critical point was determined exactly in one dimension [7]. The ferromagnetic spin-1 Ising model with biaxial CF on honeycomb lattice was studied in the EFT with correlations and the effects of the biaxial anisotropy parameters on the phase diagrams and tricritical points were investigated [8].

The ferromagnetism of spin-3/2 Ising model with transverse $\mathrm{CF}$ on honeycomb, square, and simple cubic lattices was investigated within the framework of the EFT with correlations [9]. The critical behavior and magnetic properties of the spin $3 / 2$ Blume-EmeryGriffiths model were investigated on a square lattice within the framework of EFT based on the use of a probability distribution technique and the effects of transverse $\mathrm{CF}$ on the magnetic properties were examined [10]. The ferromagnetic spin-2 Ising model with transverse CF was studied within the framework of the EFT on honeycomb, square, and cubic lattices [11]. The mixed-spin and bilayer models were also considered in the study of the transverse CF. The magnetic properties of mixed spin$1 / 2$ and spin- 1 Ising model on honeycomb lattice were investigated within the framework of a generalized startriangle mapping transformation. The particular attention was focused on the effects of uniaxial and biaxial CF anisotropies that basically influence the magnetic behavior of the spin- 1 atoms [12].

The critical behaviors of mixed spin- $1 / 2$ and $\operatorname{spin}-s_{B}$ Ising system with transverse $\mathrm{CF}$ were studied by the use of EFT with correlations and effects of the transverse CF on transition temperatures were investigated numerically for honeycomb and square lattices [13]. The phase diagrams of ferromagnetic or ferrimagnetic bilayer system consisting of two magnetic monolayers (A and B) with different spins $\left(S_{A}=1 / 2\right.$; and $\left.S_{B}=1 ; 3 / 2\right)$ and different interaction constants coupled together in a trans- 
verse $\mathrm{CF}$ were studied within the framework of the EFT with correlations [14]. Using the EFT with a probability distribution technique that accounts for the selfspin-correlation functions, critical temperature and layer longitudinal magnetizations of ferromagnetic or ferrimagnetic mixed Ising bilayer system with both spin-1/2 and spin-1 (or spin-3/2) in transverse CF were studied and their averages were examined [15].

In addition, the randomness may also change the critical behaviors of the system considerably, therefore, the random $\mathrm{CF}$ (RCF) effects on the spin models were also studied by using many approximate techniques in which the uniaxial RCF effects were mostly considered. The randomness of the transverse CF was not considered as much, i.e., only a few works can be reported. The phase transition properties of the spin-1 Blume-EmeryGriffiths model with random transverse CF were studied by the EFT on simple cubic lattice. The phase diagrams were obtained in the $T-D_{x}$ space with the ratio $\alpha$ between the biquadratic interaction and the exchange interaction as well as a tunable parameter $l$ of the transverse CF [16]. The critical properties of random transverse CF Ising model with bond dilution were studied on a square lattice with EFT. Under both weak and strong bond dilution conditions, three cases $(\alpha=0 ; \pm 0.5)$ of transverse $\mathrm{CF}$ ratio was considered in obtaining the global phase diagrams in $T-D_{x}$ space for the changes in random transverse $C F$ concentration [17]. The magnetic properties and phase diagrams of a ferrimagnetic multilayer system consisting of $L$ layers of spin-1/2 A atoms, $L$ layers of spin-1 B atoms and a disordered interface in between that is characterized by a random arrangement of $\mathrm{A}$ and $\mathrm{B}$ atoms of $\mathrm{A}_{p} \mathrm{~B}_{1-p}$ type and a negative $\mathrm{A}-\mathrm{B}$ coupling, were studied within the framework of EFT. The effects of transverse CF $D_{x}$ on the magnetic properties were examined [18]. It is clear that the random transverse $\mathrm{CF}$ effects on the spin systems have only been considered in the EFT, therefore, it is also necessary to analyze the model with some other techniques.

There are also some materials which display the evidence for the existence of the biaxial crystal fields. The biaxial CF has effects on the magnetic properties of some polymeric molecular-based magnetic materials, such as: $\mathrm{NiF}_{2}$ [19], $\mathrm{NiNO}_{3} \cdot 6 \mathrm{H}_{2} \mathrm{O}$ [20], $\mathrm{Ni}\left(\mathrm{CH}_{3} \mathrm{COO}\right)_{2} \cdot 4 \mathrm{H}_{2} \mathrm{O}$ [21], $\mathrm{Mn}\left(\mathrm{CH}_{3} \mathrm{COO}\right)_{2} \cdot 3 \mathrm{H}_{2} \mathrm{O}$ [22], $\mathrm{CoF}_{2}$ [23], $\mathrm{CoCl}_{2} \cdot 6 \mathrm{H}_{2} \mathrm{O}$ [24], and a series of compounds $\mathrm{Fe}(\mathrm{dc})_{2} \mathrm{X}[25]$, where X stands for halids and dc for the dithiocarbamate or diselenocarbamate groups, respectively (see also [12]).

Therefore, in this work, we consider the effects of random transverse CF on the spin- 1 model in the mean-field approximation (MFA). The transverse $\mathrm{CF}$ is applied to the lattice in a bimodal form. While one of the modes having the transverse $\mathrm{CF}$ values $D_{x}$, the other one is adjusted with a tunable parameter to $\alpha D_{x}$ with probabilities $p$ and $1-p$, respectively, throughout the lattice. After having obtained the order-parameters and free energy, the phase diagrams are obtained on the $\left(\Delta_{x}=D_{x} / J, k T / J\right)$ planes for constant transverse $\mathrm{CF}$ case when $q=3,4,5$ and 6 and for transverse RCF case on a square lattice $(q=4)$. The latter case was investigated in detail by varying the probability $p$ with an increment of $\Delta p=0.1$ in the range $(0,1)$ for the values of $\alpha=0, \pm 0.5$ and -1.0 . Then, the global phase diagrams on the $\left(\Delta_{x}, k T / J\right)$ planes were calculated and discussed.

The rest of this work is organized as follows. The next section is devoted to the formulation of the model in terms of the MFA. The last section includes our illustrations and findings in addition to a brief summary and conclusions.

\section{The formulation}

The Hamiltonian for the spin-1 model including the transverse RCF effects may be given as

$$
\mathcal{H}=-J \sum_{\langle i, j\rangle} S_{i}^{z} S_{j}^{z}-\sum_{i} D_{i}^{x}\left(S_{i}^{x}\right)^{2}-h \sum_{i} S_{i}^{z},
$$

where $J$ is the bilinear exchange interaction between the nearest-neighbor (NN) spins, $h$ is the longitudinal external magnetic field, $D_{i}^{x}$ is the random transverse $\mathrm{CF}$ along the $x$-axis and $\langle i, j\rangle$ indicates the summation over the NN spins. $S_{i}^{x}$ and $S_{i}^{z}$ are the components of spin-1 operator at site $i$ which are given in the matrix form as

$$
S_{i}^{x}=\frac{1}{\sqrt{2}}\left(\begin{array}{lll}
0 & 1 & 0 \\
1 & 0 & 1 \\
0 & 1 & 0
\end{array}\right) \quad \text { and } S_{i}^{z}=\left(\begin{array}{ccc}
1 & 0 & 0 \\
0 & 0 & 0 \\
0 & 0 & -1
\end{array}\right) \text {. }
$$

It is assumed that the random transverse $\mathrm{CF}$ for the site $i$ is distributed according to

$$
P\left(\Delta_{i}^{x}\right)=p \delta\left(\Delta_{i}^{x}-\Delta_{x}\right)+(1-p) \delta\left(\Delta_{i}^{x}-\alpha \Delta_{x}\right),
$$

with $\Delta_{i}^{x}=D_{i}^{x} / J$ and $\alpha$ is a tunable parameter which adjusts the strength of the transverse $\mathrm{CF}$.

Using the mean-field approximation, $\mathcal{H}$ in Eq. (1) may be put in the form

$$
-\beta \mathcal{H}_{M F A}=-\beta \sum_{i} \mathcal{H}_{M F A}^{(i)},
$$

where

$$
-\beta \mathcal{H}_{M F A}^{(i)}=\beta q J S_{z} S_{i}^{z}+\beta D_{i}^{x}\left(S_{i}^{x}\right)^{2}+\beta h S_{i}^{z},
$$

with $S_{z}=\left\langle S_{i}^{z}\right\rangle, \beta=1 /(k T), k$ is the Boltzmann constant and $q$ is the number of the NN's.

In order to calculate the components of the orderparameters, i.e. the dipolar and quadrupolar moments, the partition function is needed which is given by the definition as:

$$
\begin{gathered}
Z_{i}=\operatorname{Tr}_{(i)} \exp \left(-\beta \mathcal{H}_{M F A}^{(i)}\right)= \\
\mathrm{e}^{\lambda_{1}}+\mathrm{e}^{\lambda_{2}}+\mathrm{e}^{\lambda_{3}},
\end{gathered}
$$

where $-\beta \mathcal{H}_{M F A}^{(i)}$ can be represented with the below matrix as

$$
\begin{gathered}
-\beta \mathcal{H}_{M F A}^{(i)}= \\
\left(\begin{array}{ccc}
\beta\left(q J S_{z}+D_{i}^{x} / 2+h\right) & 0 & \beta D_{i}^{x} / 2 \\
0 & \beta D_{i}^{x} & 0 \\
\beta D_{i}^{x} / 2 & 0 & \beta\left(-q J S_{z}+D_{i}^{x} / 2-h\right)
\end{array}\right)
\end{gathered}
$$

and the eigenvalues of which are the desired $\lambda$ 's in Eq. (6). 
After a straightforward calculation of the determinant in Eq. (7), the eigenvalues are found to be

$$
\begin{aligned}
& \lambda_{1}=\beta D_{i}^{x}, \\
& \lambda_{2}=\frac{\beta}{2}\left[D_{i}^{x}+\sqrt{\left(D_{i}^{x}\right)^{2}+4\left(q J S_{z}+h\right)^{2}}\right], \\
& \lambda_{3}=\frac{\beta}{2}\left[D_{i}^{x}-\sqrt{\left(D_{i}^{x}\right)^{2}+4\left(q J S_{z}+h\right)^{2}}\right],
\end{aligned}
$$

or in terms of the reduced quantities $\beta^{\prime}=\beta J, H=h / J$ and $\Delta_{i}^{x}=D_{i}^{x} / J$, they are given as

$$
\begin{aligned}
& \lambda_{1}=\beta^{\prime} \Delta_{i}^{x}, \\
& \lambda_{2}=\frac{\beta^{\prime}}{2}\left[\Delta_{i}^{x}+\sqrt{\left(\Delta_{i}^{x}\right)^{2}+4\left(q S_{z}+H\right)^{2}}\right], \\
& \lambda_{3}=\frac{\beta^{\prime}}{2}\left[\Delta_{i}^{x}-\sqrt{\left(\Delta_{i}^{x}\right)^{2}+4\left(q S_{z}+H\right)^{2}}\right] .
\end{aligned}
$$

After having found the eigenvalues of the Hamiltonian, we can now insert them into Eq. (6) to obtain the explicit form of the partition function. Thus, the free energy can now be calculated from the partition function by using the definition $F=-(1 / \beta) \ln Z_{i}$, that is

$$
f=\frac{F}{J}=-\frac{1}{\beta^{\prime}} \ln \left[\mathrm{e}^{\lambda_{1}}+\mathrm{e}^{\lambda_{2}}+\mathrm{e}^{\lambda_{3}}\right],
$$

which will be used to obtain the order parameters of the model. Thus, the dipolar moment, or magnetization, along the $z$-axis is given in terms of the eigenvalues, i.e. Eq. (9), as

$$
\begin{aligned}
& S_{z}=\left\langle S_{i}^{z}\right\rangle=-\frac{\partial F}{\partial h}= \\
& \frac{1}{\beta} \frac{\partial \ln Z_{i}}{\partial h}=\frac{\operatorname{Tr}_{(i)}\left[S_{i}^{z} \exp \left(-\beta \mathcal{H}_{M F A}^{(i)}\right)\right]}{Z_{i}}= \\
& \frac{1}{\beta}\left[\frac{\frac{\partial \lambda_{1}}{\partial h} \mathrm{e}^{\lambda_{1}}+\frac{\partial \lambda_{2}}{\partial h} \mathrm{e}^{\lambda_{2}}+\frac{\partial \lambda_{3}}{\partial h} \mathrm{e}^{\lambda_{3}}}{\left.\mathrm{e}^{\lambda_{1}}+\mathrm{e}^{\lambda_{2}}+\mathrm{e}^{\lambda_{3}}\right]=}\right. \\
& \quad \frac{1}{\beta^{\prime}}\left[\frac{\frac{\partial \lambda_{1}}{\partial H} \mathrm{e}^{\lambda_{1}}+\frac{\partial \lambda_{2}}{\partial H} \mathrm{e}^{\lambda_{2}}+\frac{\partial \lambda_{3}}{\partial H} \mathrm{e}^{\lambda_{3}}}{\mathrm{e}^{\lambda_{1}}+\mathrm{e}^{\lambda_{2}}+\mathrm{e}^{\lambda_{3}}}\right] .
\end{aligned}
$$

Similarly, the quadrupolar order-parameter $Q_{x}$ along the $x$-axis is obtained as

$$
\begin{gathered}
Q_{x}=\left\langle\left(S_{i}^{x}\right)^{2}\right\rangle=-\frac{\partial F}{\partial D_{i}^{x}}=\frac{1}{\beta} \frac{\partial \ln Z_{i}}{\partial D_{i}^{x}}= \\
\frac{\operatorname{Tr}_{(i)}\left[\left(S_{i}^{x}\right)^{2} \exp \left(-\beta \mathcal{H}_{M F A}^{(i)}\right)\right]}{Z_{i}}= \\
\frac{1}{\beta}\left[\frac{\frac{\partial \lambda_{1}}{\partial D_{i}^{x}} \mathrm{e}^{\lambda_{1}}+\frac{\partial \lambda_{2}}{\partial D_{i}^{x}} \mathrm{e}^{\lambda_{2}}+\frac{\partial \lambda_{3}}{\partial D_{i}^{x}} \mathrm{e}^{\lambda_{3}}}{\mathrm{e}^{\lambda_{1}}+\mathrm{e}^{\lambda_{2}}+\mathrm{e}^{\lambda_{3}}}\right]= \\
\frac{1}{\beta^{\prime}}\left[\frac{\frac{\partial \lambda_{1}}{\partial \Delta_{i}^{x}} \mathrm{e}^{\lambda_{1}}+\frac{\partial \lambda_{2}}{\partial \Delta_{i}^{x}} \mathrm{e}^{\lambda_{2}}+\frac{\partial \lambda_{3}}{\partial \Delta_{i}^{x}} \mathrm{e}^{\lambda_{3}}}{\mathrm{e}^{\lambda_{1}}+\mathrm{e}^{\lambda_{2}}+\mathrm{e}^{\lambda_{3}}}\right] .
\end{gathered}
$$

In order to obtain the phase diagrams of the model at zero external magnetic field, one sets $H=0$ which leads to the spontaneous magnetization of the model. Thus, the simplified version of the magnetization at zero $H$ is found as

$$
S_{z}=\frac{4 S_{z} q}{W\left(\Delta_{i}^{x}\right)}
$$

$$
\times \frac{\mathrm{e}^{\beta^{\prime} \Delta_{i}^{x} / 2} \sinh \left(\frac{\beta^{\prime}}{2} W\left(\Delta_{i}^{x}\right)\right)}{\mathrm{e}^{\beta^{\prime} \Delta_{i}^{x}}+2 \mathrm{e}^{\beta^{\prime} \Delta_{i}^{x} / 2} \cosh \left(\frac{\beta^{\prime}}{2} W\left(\Delta_{i}^{x}\right)\right)},
$$

with $W\left(\Delta_{i}^{x}\right)=\sqrt{\left(\Delta_{i}^{x}\right)^{2}+4 q^{2} S_{z}^{2}}$. In addition, the quadrupole moment, $Q_{x}$, with $H=0$ may be simplified as

$$
\begin{aligned}
& Q_{x}=\left[\mathrm{e}^{\beta^{\prime} \Delta_{i}^{x}}+\mathrm{e}^{\beta^{\prime} \Delta_{i}^{x} / 2}\left(\cosh \left(\frac{\beta^{\prime}}{2} W\left(\Delta_{i}^{x}\right)\right)\right.\right. \\
& \left.\left.+\frac{\Delta_{i}^{x}}{W\left(\Delta_{i}^{x}\right)} \sinh \left(\frac{\beta^{\prime}}{2} W\left(\Delta_{i}^{x}\right)\right)\right)\right] / \\
& {\left[\mathrm{e}^{\beta^{\prime} \Delta_{i}^{x}}+2 \mathrm{e}^{\beta^{\prime} \Delta_{i}^{x} / 2} \cosh \left(\frac{\beta^{\prime}}{2} W\left(\Delta_{i}^{x}\right)\right)\right] .}
\end{aligned}
$$

After having calculated the free energy, the magnetization along the $z$-axis and quadrupolar order parameter along the $x$-axis, we are now ready to numerically study their temperature variations to obtain the critical behaviors of the random transverse $\mathrm{CF}$ on the spin-1 system. Note again that the CF distribution given in Eq. (3) must be applied to the above equations to include the effects of randomness of the CF into the model which is done by employing the standard random case [26]. It should also be mentioned that the mean-field equations are calculated iteratively and in each step of calculation Eq. (3) is applied which alters the mean-field equations including the random transverse $\mathrm{CF}$ effects.

\section{Results and conclusions}

In this section, the phase diagrams are illustrated on the $\left(\Delta_{x}, k T / J\right)$ planes for the case with constant transverse $\mathrm{CF}$, i.e. $p=1.0$, and the random transverse $\mathrm{CF}$ case by varying the probability or the concentration $p$ in the range $(0,1)$ with an increment of $\Delta p=0.1$ when the tunable parameter $\alpha$ is set to the values $0.0, \pm 0.5$ and -1.0 for $q=4$, i.e. on a square lattice. In the phase diagrams, the second- and first-order phase transition lines are shown with the solid and dashed lines, respectively. The tricritical, critical end points, and end points are labeled with the triangles, circles, and stars, respectively.

Before illustrating the phase diagrams, we have to explain the procedure of finding the phase transition temperatures and special points. As the temperature is increased, the magnetization goes to zero continuously separating the ferromagnetic phase from the paramagnetic one at the second-order phase transition temperature $T_{\mathrm{c}}$ where the quadrupolar moment exhibits a cusp and the free energy is single-valued. At the first-order phase transition temperature, $T_{\mathrm{t}}$, the order-parameters present jump discontinuities and the free energy is multivalued. The phase region is ferromagnetic below each line and paramagnetic above each line. The tricritical point (TCP) is the point at which the $T_{\mathrm{c}}$-line terminates from where the $T_{\mathrm{t}}$-line emerges and at the critical end point the $T_{\mathrm{c}}$-line intersects with the $T_{\mathrm{t}}$-line.

Figure 1 is obtained for $p=1$ which corresponds to constant CF case. The second-order lines, or the $T_{\mathrm{c}}$-lines, appear at negative $\Delta_{x}$ when $k T / J=0.0$. Their temperatures increase very sharply with the very little increase of $\Delta_{x}$ at its lowest negative values. As $\Delta_{x}$ increases fur- 


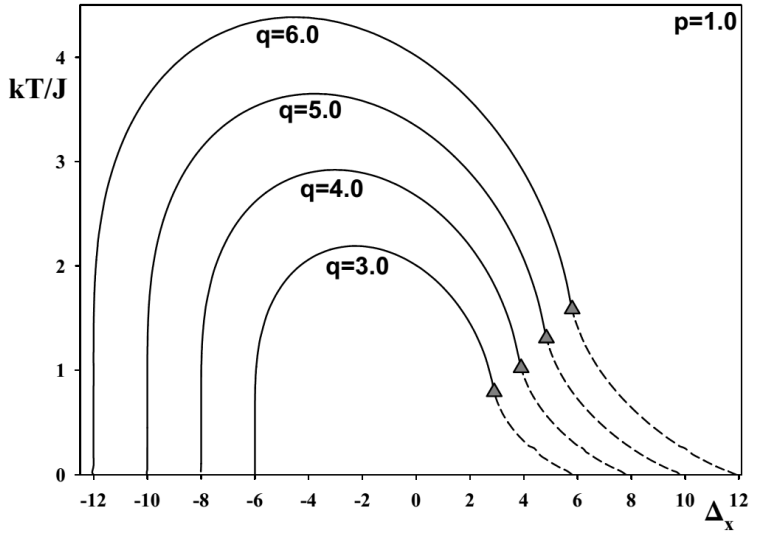

Fig. 1. The phase diagrams on the $\left(\Delta_{x}, k T / J\right)$ plane for the constant transverse CF case $(p=1.0)$ when $q=$ $3,4,5$ and 6. ther, the $T_{\mathrm{c}}$-lines make almost half circles. With the further increase of $\Delta_{x}$, the $T_{\mathrm{c}}$-lines terminate at lower temperatures at the tricritical points (TCP) from where the first-order phase transitions lines, i.e. $T_{\mathrm{t}}$-lines, emerge. They gradually decrease in temperature and disappear at higher positive $\Delta_{x}$ values at zero $k T / J$. It is clear that all the critical lines have similar shapes for each $q$, but their temperatures increase as $q$ increases. The TCP's are also seen at higher $k T / J$ 's for higher $q$ 's. It should be noted that the shapes of these critical lines are very similar with Fig. 1 of [4] and Fig. 1a of [6] where the lines are only the $T_{\mathrm{c}}$-lines with no TCP's. In addition, the temperatures of these lines are higher and $\Delta_{x}$ extends further in the range for the MFA, i.e. in this work. Note that the existence of TCP of this work is in agreement with Fig. 1b of [5] and the shape of critical line is also very similar.
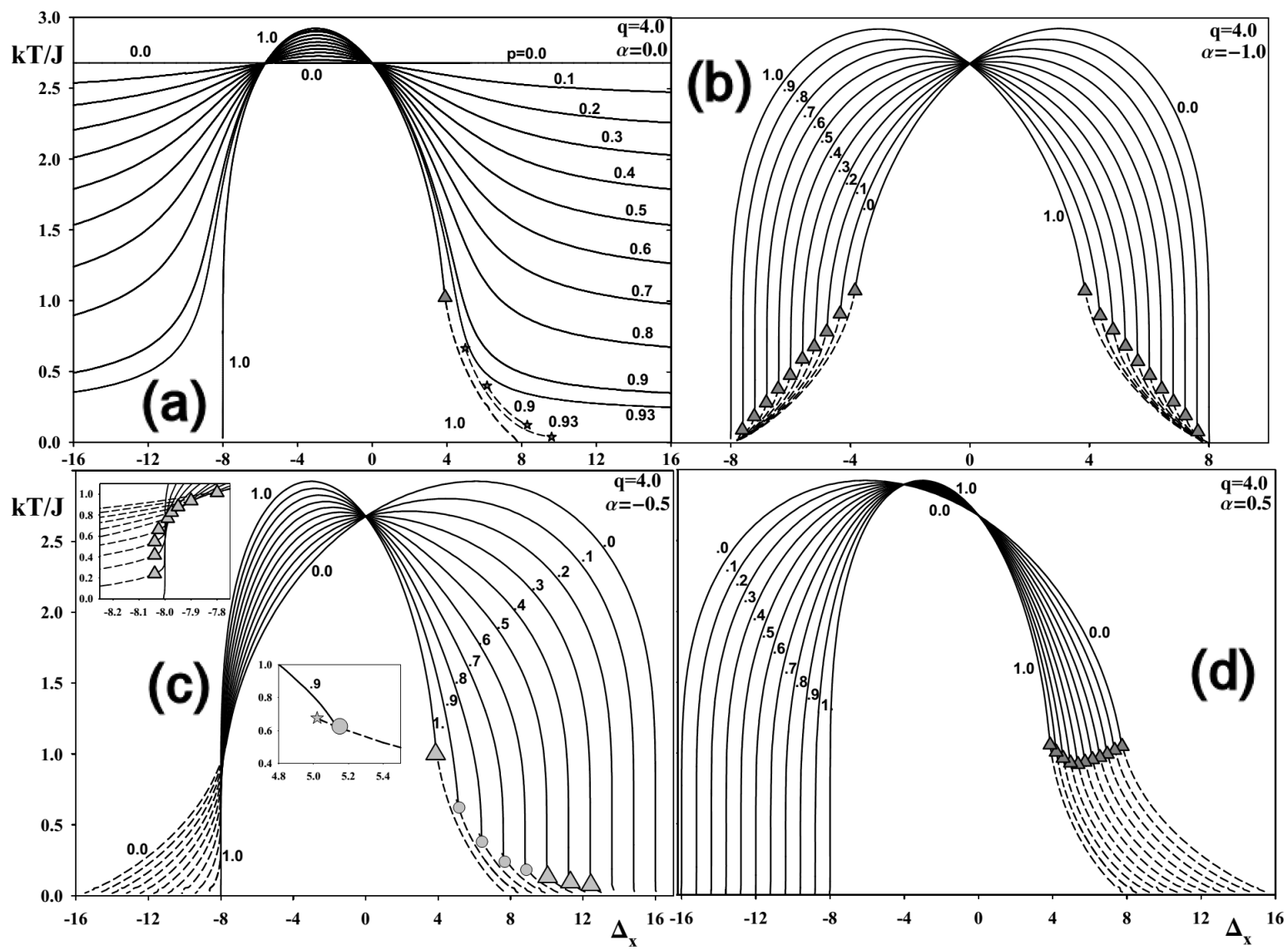

Fig. 2. The phase diagrams on the $\left(\Delta_{x}, k T / J\right)$ planes for the random transverse CF case when $p$ is varied between 0.0 and 1.0 with the increment of $\Delta p=0.1$ when $q=4$ for (a) $\alpha=0.0$ ( $p=0.93$ was also included in this case), (b) $\alpha=-1.0$, (c) $\alpha=-0.5$ and (d) $\alpha=0.5$.

Figure 2 is obtained for the random transverse $\mathrm{CF}$ case. Again the phase diagrams are calculated on the $\left(\Delta_{x}, k T / J\right)$ planes with the values of the tunable parameter are set to $\alpha=0.0, \pm 0.5$ and -1.0 in Eq. (3) and where the lines are labeled with the values of $p$ :

(i) In Fig. 2a, $\alpha$ is set to zero. When $p=0.0$, the crystal field is turned off for all the sites on the lattice, thus only the first term of the Hamiltonian remains. As a result of this independence from the CF, the system gives a straight line in the form of a $T_{\mathrm{c}}$-line as expected. When $0.1 \leq p \leq 0.9$ and $p=0.93$, the $T_{\mathrm{c}}$-lines has three portions: the left and right wings decrease in temperature as $p$ increases and they reach to constant temperatures as $\left|\Delta_{x}\right|$ becomes large and in the middle portion the 
temperatures of the $T_{\mathrm{c}}$-lines increase as $p$ increases. All the lines intersect at two special points with $\Delta_{x}=0.0$, which correspond to $p=1.0$ case, and $\Delta_{x} \cong-5.7$ at $k T / J=2.678$. When $p=1.0$, we see that the $T_{\mathrm{c}}$-line goes to zero at about $\Delta_{x} \cong-8.0$, TCP is about $\Delta_{x} \cong 3.9$ and $k T / J \cong 1.023$ and the $T_{\mathrm{t}}$-line terminates at $\Delta_{x} \cong 8.0$ which is the same plot as in Fig. 1. A portion of $T_{\mathrm{t}}$ line without connection to anywhere is also found when $p=0.9$ and 0.93 . This may be such that the $T_{\mathrm{t}}$-line of the case with $p=1.0$ is broken from the TCP and becomes shorter as $p$ gets smaller in the figure with $p=0.9$ and 0.93 . It should also be mentioned that the magnetization gives two phase transitions for the appropriate values of the system parameters: $T_{\mathrm{t}}$ at lower temperatures and $T_{\mathrm{c}}$ at higher temperatures for $p=0.9$ and 0.93 , therefore, their lines also separate the ferromagnetic phases from paramagnetic phases in the different regions of the phase diagrams.

(ii) In Fig. 2b, $\alpha=-1.0$. The critical lines completing each other to 1.0 are symmetrical, such as $(0.0,1.0),(0.1$, $0.9)$, etc. This is expected as seen from Eq. (3). When $p=0.0$ and 1.0, there is only one TCP for each case. But for the interval values, there are two TCP's. For negative $\Delta_{x}$ 's, the TCP's are seen at lower temperatures for higher $p$ values. But for positive $\Delta_{x}$ 's, it occurs the other way around. In this case, the $T_{\mathrm{c}}$-lines coincide only once at $\Delta_{x}=0.0$.

(iii) $\alpha=-0.5$ in Fig. 2c. Again $p=1.0$ case is the same as before. For negative $\Delta_{x}$, the model exhibits both $T_{\mathrm{c}^{-}}$ and $T_{\mathrm{t}}$-lines meeting at the TCP's for $0.1 \leq p \leq 0.9$ as seen from the figure. As $p$ increases, the places of the TCP's move towards right and upward. When $\Delta_{x}$ is positive, we see that the model does not display any TCP's for $p=0.0,0.1$ and 0.2 , but the TCP's are seen for $p=0.3,0.4,0.5$ and 1.0. In addition, the critical end points (see inset when $p=0.9$ for example) and end points of the $T_{\mathrm{t}}$-lines are also seen when $p=0.6,0.7,0.8$ and 0.9 . The latter two points have not yet been reported in any other works as far as in our knowledge.

(iv) $\alpha=0.5$ case is illustrated in Fig. $2 \mathrm{~d}$. Only the $T_{\mathrm{c}^{-}}$ lines for the negative $\Delta_{x}$ and the combinations of the $T_{\mathrm{c}^{-}}$ and $T_{\mathrm{t}}$-lines meeting at the TCP's for the positive $\Delta_{x}$ are found. The TCP start from higher temperatures when $p=1.0$ and its temperatures decrease as $p$ decreases, then it starts increasing with further decrease of $p$. Again the $T_{\mathrm{c}}$-lines cross each other at two special points at $\Delta_{x} \cong$ -4.02 and $k T / J \cong 2.89$ and at $\Delta_{x}=0.0$ and $k T / J \cong$ 2.678 .

In conclusion, the effects of the random transverse $\mathrm{CF}$ was investigated in the MFA on the spin- 1 system. Its effects were examined on the phase diagrams by varying the probability $p$ on a square lattice. It was found that the model gives both second- and first-order phase transitions and thus TCP's. In addition, it was also found that the model also yields critical end points and the end points for the first-order phase transition lines for appropriate values of $p$ which was not observed in the previous works i.e. $[16,17]$. The $T_{\mathrm{t}}$ tail is short as seen in the inset of Fig. 2c that is why its existence might have escaped from the eye in $[16,17]$. Clearly, the transverse CF and its randomness affect the critical behaviors of the model considerably. The existence of the special points where all the $T_{\mathrm{c}}$-lines intersect other than $\Delta_{x}=0.0$ may have a special interpretation and usage.

\section{References}

[1] G.P. Taggart, R.A. Tahir-Kheli, E. Shiles, Physica 75, 234 (1974).

[2] R. Micnas, Physica A 89, 431 (1977).

[3] K.K. Pan, Y.L. Wang, Phys. Lett. A 178, 325 (1993); ibid., Phys. Rev. B 51, 3610 (1995).

[4] N.C. Eddeqaqi, M. Saber, A. El-Atri, M. Kerouad, Physica A 272, 144 (1999).

[5] J.R. de Sousa, N.S. Branco, Phys. Rev. E 77, 012104 (2008).

[6] S. Bouhou, I. Essaoudi, A. Ainane, M. Saber, M. Kerouad, F. Dujardin, J. Supercond. Nov. Magn. 24, 571 (2011).

[7] J. Oitmaa, A.M.A. von Brasch, Phys. Rev. B 67, 172402 (2003).

[8] Q. Zhang, G.-Z. Wei, Y.-Q. Liang, J. Magn. Magn. Mater. 253, 45 (2002).

[9] W. Jiang, G.-Z. Wei, A. Du, L.-Q. Guo, Physica A 313, 503 (2002).

[10] M. Boughrara, M. Kerouad, M. Saber, Chin. J. Phys. 49, $672(2011)$.

[11] W. Jiang, G.-Z. Wei, Z.-H. Xin, Phys. Status Solidi B 221, 759 (2000).

[12] J. Strečka, M. Jaščur, Phys. Rev. B 70, 014404 (2004).

[13] W. Jiang, G.-B. Xiao, G.-Z. Wei, D. An, Z. Qi, Commun. Theor. Phys. 41, 131 (2004).

[14] W. Jiang, G.-Z. Wei, A. Du, J. Magn. Magn. Mater. 250, 49 (2002).

[15] K. Htoutou, A. Ainane, M. Saber, J. Magn. Magn. Mater. 269, 245 (2004).

[16] X. Qian, S.L. Yan, Solid State Commun. 151, 1846 (2011).

[17] W. Ling, S.-L. Yan, Cent. Eur. J. Phys. 9, 1084 (2011).

[18] M. Boughrara, M. Kerouad, Physica A 374, 669 (2007).

[19] T. Moriya, Phys. Rev. 117, 635 (1960).

[20] L. Berger, S.A. Friedberg, Phys. Rev. 136, A158 (1964).

[21] L.G. Polgar, S.A. Friedberg, Phys. Rev. B 6, 3497 (1972).

[22] H. Kumagai, K. Ôno, I. Hayashi, K. Kambe, Phys. Rev. 87, 374 (1952).

[23] M.E. Lines, Phys. Rev. 137, A982 (1965).

[24] N. Uryû, J. Skalyo, S.A. Friedberg, Phys. Rev. 144, 689 (1966).

[25] G.C. DeFotis, F. Palacio, R.L. Carlin, Phys. Rev. $B$ 20, 2945 (1979); G.C. DeFotis, B.K. Failon, F.V. Wells, H.H. Wickman, ibid., 29, 3795 (1984).

[26] E. Albayrak, Solid State Commun. 159, 76 (2013). 